\title{
One-Photon Solutions to the Multiqubit Multimode Quantum Rabi Model for Fast $W$-State Generation
}

\author{
Jie Peng $\odot,{ }^{1, *}$ Juncong Zheng $\odot,{ }^{1}$ Jing Yu, ${ }^{2}$ Pinghua Tang, ${ }^{1}$ G. Alvarado Barrios, ${ }^{2}$ Jianxin Zhong, ${ }^{1}$ Enrique Solano, ${ }^{2,3,4,5, \uparrow}$ \\ F. Albarrán-Arriagada $\oplus^{2}$ and Lucas Lamata $\oplus^{6, \ddagger}$ \\ ${ }^{1}$ Hunan Key Laboratory for Micro-Nano Energy Materials and Devices and School of Physics and Optoelectronics, \\ Xiangtan University, Hunan 411105, China \\ ${ }^{2}$ International Center of Quantum Artificial Intelligence for Science and Technology (QuArtist) and \\ Physics Department, Shanghai University, 200444 Shanghai, China \\ ${ }^{3}$ Department of Physical Chemistry, University of the Basque Country UPV/EHU, Apartado 644, 48080 Bilbao, Spain \\ ${ }^{4}$ IKERBASQUE, Basque Foundation for Science, Plaza Euskadi 5, 48009 Bilbao, Spain \\ ${ }^{5}$ Kipu Quantum, Kurwenalstrasse 1, 80804 Munich, Germany \\ ${ }^{6}$ Departamento de Física Atómica, Molecular y Nuclear, Universidad de Sevilla, 41080 Sevilla, Spain
}

(Received 3 March 2021; accepted 17 June 2021; published 22 July 2021)

\begin{abstract}
General solutions to the quantum Rabi model involve subspaces with an unbounded number of photons. However, for the multiqubit multimode case, we find special solutions with at most one photon for an arbitrary number of qubits and photon modes. Such solutions exist for arbitrary single qubit-photon coupling strength with constant eigenenergy, while still being qubit-photon entangled states. Taking advantage of their peculiarities and the reach of the ultrastrong coupling regime, we propose an adiabatic scheme for the fast and deterministic generation of a two-qubit Bell state and arbitrary single-photon multimode $W$ states with nonadiabatic error less than $1 \%$. Finally, we propose a superconducting circuit design to catch and release the $W$ states, and shows the experimental feasibility of the multimode multiqubit quantum Rabi model.
\end{abstract}

DOI: 10.1103/PhysRevLett.127.043604

Introduction.-The quantum Rabi model [1,2] describes the interaction between a two-level system and a single photonic mode at the most fundamental level. Since it involves both rotating and counterrotating interaction terms, all Fock states are connected and there is no closed subspace, turning the Hamiltonian hard to solve. Therefore, despite its simple form, the exact solution of the model was not found until 2011 [3], invoking a lot of interest in this model [4-13] thereafter.

The Rabi model plays an important role in quantum optics [14-17], optomechanics [18], condensed matter physics [19], and so on [20-22]. However, in most applications we need to consider more than one qubit and/or one mode, e.g., to perform a controlled gate [23,24] for universal quantum computing $[25,26]$, and to generate multipartite entangled states [27-29]. Hence, a mathematical description with physical implications for the multiqubit multimode quantum Rabi model (MMQRM) is essential for the development of scalable and efficient protocols, suitable for current technology demands.

In this Letter, we find special solutions with at most one photon to the MMQRM for arbitrary number of qubits and modes, although the interaction terms still connect all photon number states. Unlike Judd's isolated quasiexact solutions (exact solutions for part of the spectrum [30,31]) to the quantum Rabi model [32], these solutions exist for any couplings with constant energy when qubit and mode frequencies are fixed, provided the couplings themselves satisfy certain condition. They are a coherent superposition of qubit-photon states, which makes the photon population trapped in zero and one, and we call it a special dark state [33]. Furthermore, we use such solutions to propose a fast and deterministic entangled state generation protocol to obtain a two-qubit Bell state and an arbitrary single-photon $M$-mode $W$ state $|W\rangle_{M}=$ $(1 / \mathcal{N}) \sum_{i=1}^{M} g_{i}\left|0_{1} 0_{2} \cdots 1_{i} 0_{i+1} \cdots 0_{M}\right\rangle$ [34-37] simultaneously through adiabatic passage. Here, $g_{i}$ is the coupling strength between the qubits and $i$ th photon mode. It is known that $W$ states are robust under particle loss [38] and a central resource in several quantum information processing protocols [39-42]. Consequently, various schemes have been presented to generate them [43-54]. Because of the reach of ultrastrong coupling and peculiarities of the special dark states, the most interesting advantage of using the MMQRM is the fast generation (less than $70 \omega^{-1}$ ) and low nonadiabatic error (less than $1 \%$ ), outperforming the recently reported fastest two-qubit CPHASE gate (30-4-5 ns) [55] for photon frequency $\omega / 2 \pi=3 \mathrm{GHz}$. The generation time is almost the same for different mode numbers and no external laser is needed. Since $g_{i}$ is adjustable, we can generate any $W$ state in a unified and convenient way. Finally, we propose a superconducting circuit design for the 
MMQRM, as well as for the catch and release of these $W$ states. These results pave the way to the implementation of fast protocols in quantum information using the MMQRM.

Special quasiexact solutions to the MMQRM.-We present our method to obtain the quasiexact solutions with at most one photon to the MMQRM

$H=\sum_{i=1}^{M} \omega_{i} a_{i}^{\dagger} a_{i}+\sum_{i=1}^{M} \sum_{j=1}^{N} g_{i j} \sigma_{j x}\left(a_{i}+a_{i}^{\dagger}\right)+\sum_{j=1}^{N} \Delta_{j} \sigma_{j z}$,

where $a_{i}^{\dagger}$ and $a_{i}$ are the $i$ th photon mode creation and annihilation operators with frequency $\omega_{i}$, respectively. Also, $\sigma_{j \alpha}(\alpha=x, y, z)$ are the Pauli matrices corresponding to the $j$ th qubit, $2 \Delta_{j}$ is the energy level splitting of the $j$ th qubit, and $g_{i j}$ is the qubit-photon coupling parameter between the $i$ th mode and $j$ th qubit.

Since Hamiltonian (1) breaks the $U(1)$ symmetry, there is no closed subspace consisting of finite photon number states. However, it has a $\mathbb{Z}_{2}$ symmetry with generator $R=\exp \left[i \pi \sum_{i=1}^{M} a_{i}^{\dagger} a_{i}\right] \Pi_{j} \sigma_{j z}$. Accordingly, we categorize all $N$-qubit states $\left(\left|\psi_{N q}\right\rangle\right)$ into two sets corresponding to the eigenvalues of $\Pi_{j} \sigma_{j z}$ being \pm 1 , and denote them $2^{N-1}$ dimensional row vectors, $\left(\left|\psi_{N q, \pm}\right\rangle\right)$. We also denote all $k$ photon states with $M$ modes by $\left(\left|k_{M}\right\rangle\right)$. Hence, there are two invariant subspaces

$$
\begin{aligned}
& \left(\left|0_{M}, \psi_{N q,+}\right\rangle\right) \leftrightarrow\left(\left|1_{M}, \psi_{N q,-}\right\rangle\right) \leftrightarrow\left(\left|2_{M}, \psi_{N q,+}\right\rangle\right) \cdots, \\
& \left(\left|0_{M}, \psi_{N q,-}\right\rangle\right) \leftrightarrow\left(\left|1_{M}, \psi_{N q,+}\right\rangle\right) \leftrightarrow\left(\left|2_{M}, \psi_{N q,-}\right\rangle\right) \cdots,
\end{aligned}
$$

with positive and negative parity, respectively. So $H$ will take the following form in the \pm parity subspace

$$
H^{ \pm}=\left(\begin{array}{cccccc}
D_{0}^{ \pm} & O_{0}^{\dagger} & 0 & 0 & 0 & \ldots \\
O_{0} & D_{1}^{ \pm} & O_{1}^{\dagger} & 0 & 0 & \ldots \\
0 & O_{1} & D_{2}^{ \pm} & O_{2}^{\dagger} & 0 & \ldots \\
\ldots & \ldots & \ldots & \ldots & \ldots & \ldots
\end{array}\right)
$$

where $D_{k}^{ \pm}$is a $2^{N-1} C_{M+k-1}^{k} \times 2^{N-1} C_{M+k-1}^{k}$ matrix and $O_{k}$ is a $2^{N-1} C_{M+k}^{k+1} \times 2^{N-1} C_{M+k-1}^{k}$ matrix which takes the same form for \pm parity [56]. Although all Fock states are connected, it is possible to find solutions with finite photon numbers. Indeed, there are such solutions in the singlemode multiqubit quantum Rabi model [57-59].

We search now for solutions with at most $L$ photons taking the form $\left|\psi_{ \pm}\right\rangle=\vec{c}_{0 M}^{ \pm}\left(\left|0_{M}, \psi_{N q, \pm}\right\rangle\right)+$ $\vec{c}_{1 M}^{ \pm}\left(\left|1_{M}, \psi_{N q, \mp}\right\rangle\right)+\cdots+\vec{c}_{L M}^{ \pm}\left(\left|L_{M}, \psi_{N q, \pm(-1)^{L}}\right\rangle\right)$ to the MMQRM, where $\vec{c}_{k, M}^{ \pm}$is a $2^{N-1} C_{M+k-1}^{k}$ dimensional vector since $\left(\left|k_{M}, \psi_{N q, \pm(-1)^{k}}\right\rangle\right)$ is a vector of the same dimension, by solving the eigenenergy equation

$$
\left(H^{ \pm}-E^{ \pm}\right)\left|\psi_{ \pm}\right\rangle=0 .
$$

According to Eqs. (4) and (5), there are more equations than variables $\vec{c}_{k, M}^{ \pm}$since we do not truncate $H$. But quasiexact solutions could exist when parameters meet a certain condition. For $L>1$ this condition reads $f\left(g_{i j}, \omega_{i}, \Delta_{j}\right)=0$ [56], just like Judd's solution [32] to the quantum Rabi model. For $L=1$, it reduces to $f^{\prime}\left(\omega_{i}, \Delta_{j}\right)=0$ and $f^{\prime \prime}\left(g_{i j}\right)=0$, so the solutions change from isolated points into lines in the spectra for certain $\omega_{i}$ and $\Delta_{j}$. The latter may have important applications in quantum information, as will be discussed below. Hence, we will focus on $L=1$, where Eq. (5) reduces to

$$
\left(\begin{array}{cc}
D_{0}^{ \pm}-E^{ \pm} & 0 \\
O_{0} & D_{1}^{ \pm}-E^{ \pm} \\
0 & O_{1}
\end{array}\right)\left(\begin{array}{l}
\vec{c}_{0_{M}}^{ \pm} \\
\vec{c}_{1_{M}}^{ \pm}
\end{array}\right)=0
$$

after matrix elementary row transformation [56]. For the single qubit and single-mode case, $O_{1}$ is just a $c$ number, so there is no nontrivial solution for $O_{1} \vec{c}_{11}^{ \pm}=0$. However, for the multiqubit case, it can be satisfied if matrix $O_{1}$ has eigenvalue 0 with $\vec{c}_{1 M}^{ \pm}$being its corresponding eigenvector. Then, we make elementary row transformations to the matrix in Eq. (6), and the solution is obtained if there are more columns than nonzero rows. At the same time, the condition for the parameters is calculated.

For the two-qubit and $M$-mode case, we find the special solution for even parity to be [56]

$\left|\psi_{2+}\right\rangle=\frac{1}{\mathcal{N}}\left[\left(\Delta_{1}-\Delta_{2}\right)\left|0_{M}, \uparrow, \uparrow\right\rangle+\left|W_{M}\right\rangle(|\downarrow, \uparrow\rangle-|\uparrow, \downarrow\rangle)\right]$,

where $\left|W_{M}\right\rangle=g_{1}|1,0,0, \ldots, 0\rangle+g_{2}|0,1,0, \ldots, 0\rangle+\cdots+$ $g_{M}|0,0,0, \ldots, 1\rangle$ with the condition $\omega_{i}=\omega$ for all $i, g_{i j}=$ $g_{i}$ for all $j$ and $\Delta_{1}+\Delta_{2}=\omega=E^{+}$. This is a special dark state with at most one photon, which exists for arbitrary $g_{i}$ with constant eigenenergy $E^{+}=\omega$, corresponding to a horizontal line in the spectrum [see Fig. 1(a)], while still being a qubit-photon entangled state. There are two similar solutions $\left|\psi_{2-}\right\rangle$ corresponding to odd parity [56].

For the three-qubit and $M$-mode case, the quasiexact solution for odd parity reads

$$
\begin{aligned}
\left|\psi_{3-}\right\rangle= & \left|W_{M}\right\rangle(|\uparrow, \downarrow, \downarrow\rangle-|\downarrow, \uparrow, \downarrow\rangle-|\downarrow, \downarrow, \uparrow\rangle+|\uparrow, \uparrow, \uparrow\rangle) \\
& +\frac{\omega g_{13}}{g_{12}}\left|0_{M}, \uparrow, \uparrow, \downarrow\right\rangle+\frac{\omega g_{12}}{g_{13}}\left|0_{M}, \uparrow, \downarrow, \uparrow\right\rangle \\
& -\frac{\omega g_{11}^{2}}{g_{12} g_{13}}\left|0_{M}, \downarrow, \uparrow, \uparrow\right\rangle,
\end{aligned}
$$

where $\left|W_{M}\right\rangle=g_{11}|1,0,0, \ldots, 0\rangle+g_{21}|0,1,0, \ldots, 0\rangle+\cdots+$ $g_{M 1}|0,0,0, \ldots, 1\rangle$ with the conditions $\Delta_{j}=\omega_{i}=\omega=E^{-}$, 

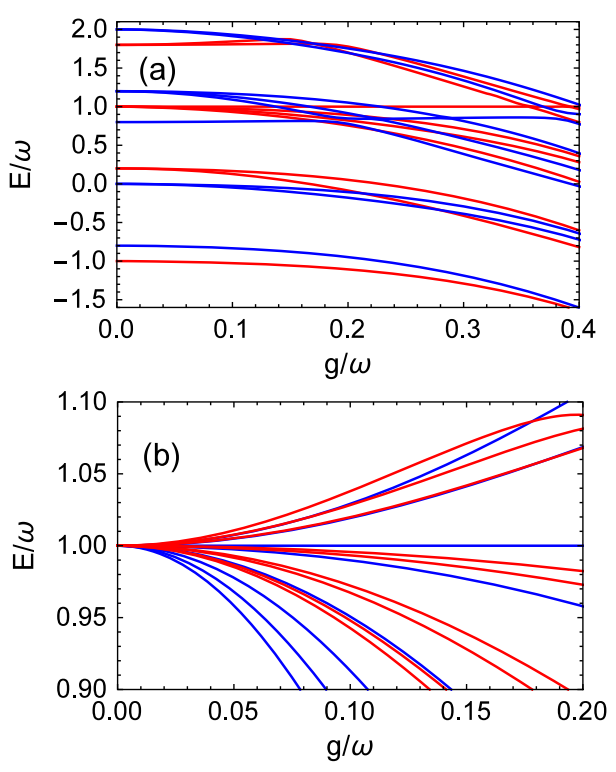

FIG. 1. (a) Spectrum of the two-qubit two-mode quantum Rabi model with $\omega_{1}=\omega_{2}=\omega, \Delta_{1}=0.9 \omega, \Delta_{2}=0.1 \omega, g_{11}=g_{12}=$ $g_{21}=g_{22}=g$. (b) Spectrum of the three-qubit two-mode quantum Rabi model with $\Delta_{1}=\Delta_{2}=\Delta_{3}=\omega_{1}=\omega_{2}=\omega$, $g=g_{11}=g_{12}+g_{13}=g_{21}, g_{12}=g_{22}=g_{13}=g_{23}$. Red lines correspond to even parity while blue lines to odd parity.

$g_{i 1}=g_{i 2}+g_{i 3}$. This eigenstate corresponds to the horizontal line $E / \omega=1$ in Fig. 1(b).

For the $N$-qubit and $M$-mode case, there exist special dark states with $E=\omega[56]$

$$
\begin{aligned}
& \left|\psi_{N}\right\rangle=\left|\psi_{2}\right\rangle \otimes\left(\left|\psi_{B}\right\rangle\right)^{(N-2) / 2}, \quad N=2,4,6,8, \ldots, \\
& \left|\psi_{N}\right\rangle=\left|\psi_{3}\right\rangle \otimes\left(\left|\psi_{B}\right\rangle\right)^{(N-3) / 2}, \quad N=3,5,7,9, \ldots,
\end{aligned}
$$

where $\left|\psi_{B}\right\rangle=(1 / \sqrt{2})(|\downarrow \uparrow\rangle-|\uparrow \downarrow\rangle)$ is the two-qubit singlet Bell state and $\left|\psi_{2,3}\right\rangle$ includes $\left|\psi_{2 \pm}\right\rangle$ and $\left|\psi_{3-}\right\rangle$, which has been obtained above.

Fast generation of the arbitrary single-photon multimode $W$ state.-Here, the special dark state $\left|\psi_{2+}\right\rangle$ Eq. (7) is an excellent candidate for deterministically generating arbitrary $W$ states $\left|W_{M}\right\rangle$ through adiabatic passage: (1) The initial state $\left|0_{M}, \uparrow, \uparrow\right\rangle$ is easy to prepare, and the target state is obtained once $\Delta_{1}-\Delta_{2}=0$ for any nonzero $g_{i}$ with no external laser needed. (2) The coefficient of $\left|0_{1} 0_{2} \cdots 1_{i} 0_{i+1} \cdots 0\right\rangle$ is just $g_{i}$ in $\left|W_{M}\right\rangle$, so any $W$ state can be generated by adjusting $g_{i}$ directly.

Our scheme is as follows. First, two qubits are excited by pumping pulses and coupled to $M$ resonators in vacuum states with initial coupling strength $g_{i 1}=g_{i 2}=g_{i}=0$. The qubit frequencies are nonidentical and always satisfy $\Delta_{1}+\Delta_{2}=\omega_{i}=\omega$. Then, we slowly decrease $\left|\Delta_{1}-\Delta_{2}\right|$ to 0 while we increase $g_{i}$ to a nonzero value, so that the target state $\left|W_{M} \psi_{B}\right\rangle$ is obtained. As an example, the
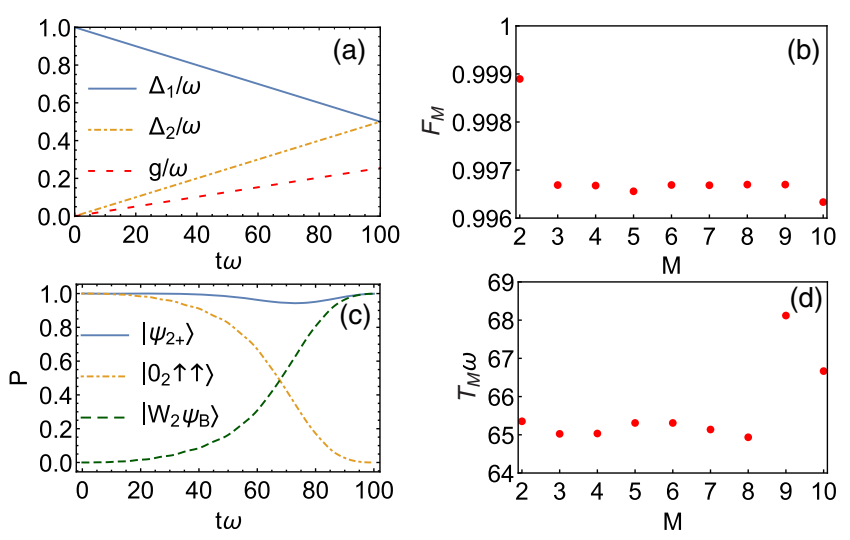

FIG. 2. Adiabatic evolution of the special dark state $\left|\psi_{2+}\right\rangle$ of Eq. (7) from $\left|0_{M} \uparrow \uparrow\right\rangle$ to $\left|W_{M} \psi_{B}\right\rangle$ for the two-qubit $M$-mode quantum Rabi model, where $\left|\psi_{B}\right\rangle=(1 / \sqrt{2})(|\downarrow \uparrow\rangle-|\uparrow \downarrow\rangle)$, and $\left|W_{M}\right\rangle$ is the normalized prototype $W$ state with all $g_{i}$ 's equal. (a) Adiabatic trajectory used to vary the parameters for $M=2$. $g_{1}=g_{2}=g$. (b) Fidelity $F_{M}=\left|\left\langle W_{M} \psi_{B} \mid \psi(T)\right\rangle\right|^{2}$ when $T$ is fixed to $100 \omega^{-1}$ for mode number $M$. (c) Population of different states during the adiabatic process for $M=2$. (d) Interaction time needed to reach $F_{M}>0.99$ for mode $M$ in unit of $\omega^{-1}$.

numerical simulation for the adiabatic evolution of the two-qubit two-mode case is shown in Fig. 2(c) with the adiabatic trajectory used to vary the parameters depicted in Fig. 2(a). The evolution time is just $T=100 \omega^{-1}$ and the fidelity $F_{2}=\left|\left\langle\psi(T) \mid W_{2} \psi_{B}\right\rangle\right|^{2}$ reaches $99.89 \%$. If the evolution time is fixed to $100 \omega^{-1}$, the fidelities $F_{M}$ for the $M$-mode case are shown in Fig. 2(b), which are almost equal and higher than $99.6 \%$. If the fidelities are restricted to be higher than $99 \%$, the time costs for each mode are shown in Fig. 2(d), which are less than $69 \omega^{-1}$. The linear adiabatic trajectories for different cases are shown in [56].

According to the current available circuit QED technology, the transmon frequency $\Delta / \pi$ can be tuned from 0 to $6 \mathrm{GHz}[60,61]$, hence the resonator frequency $\omega / 2 \pi$ is chosen to be $3 \mathrm{GHz}$ [62] to satisfy $\Delta_{1} \pm \Delta_{2}=\omega$ at $g_{i} / 2 \pi=0$. The couplings could satisfy $g_{i 1}=g_{i 2}$ in experiments $[63,64]$. They are also tunable $[64,65]$, even independently of qubit frequencies [66], or from 0 to half of the resonator frequency [23]. Therefore, our scheme shown in Fig. 2(a) is within experimental reach and the adiabatic evolution takes only $33.3 \mathrm{~ns}$ with nonadiabatic error $0.1 \%$. It is faster than the current state-of-the-art two-qubit CPHASE gate with an operation time of $40 \mathrm{~ns}[67,68]$, and comparable to recently reported fastest two-qubit gates of 30-45 ns [55]. If we restrict the nonadiabatic error to less than $1 \%$, then the average evolution time to generate $\left|W_{M} \psi_{B}\right\rangle$ from $\left|0_{M} \uparrow \uparrow\right\rangle$ for $M=2,3, \ldots, 10$ will be 21.9 ns. Note that we have chosen the simplest linear adiabatic path shown in Fig. 2(a), but we can also consider a "faster adiabatic" trajectory to reduce the time such as in Refs. $[67,69,70]$. 
The fast adiabatic speed is due to the reach of ultrastrong coupling and the peculiarities of the special dark state $\left|\psi_{2+}\right\rangle$ [56].

(1) $\left\langle\psi_{E=\omega}|\dot{H}| \psi_{2+}\right\rangle=0$, no matter how fast the parameters change, so that the adiabatic speed is not limited by the vanishing energy gap at the degeneracy points [e.g., around $g / \omega \approx 0.357$ and 0.392 in Fig. 1(a)] according to the adiabatic theorem $[71,72]$

$\left|\frac{\left\langle E_{m}(t)|\dot{H}| E_{n}(t)\right\rangle}{\left(E_{m}-E_{n}\right)^{2}}\right| \ll 1, \quad m \neq n, \quad t \in[0, T]$.

(2) There are $C_{M+1}^{2}+1$ degenerate eigenstates $\left|\psi_{E=\omega}\right\rangle$ at the Jaynes-Cummings coupling regime when the rotating wave approximation is applied. They reduce to very close eigenstates in the MMQRM spectrum at this regime, as shown in Fig. 1(a) for $M=2$. We name them $\left|\psi_{\omega_{1,2,3,4}}\right\rangle$ in energy descending order with $\left|\psi_{\omega_{1}}\right\rangle=\left|\psi_{2+}\right\rangle$. Numerical results show $\left\langle\psi_{\omega_{2,3}}|\dot{H}| \psi_{2+}\right\rangle=0$, where $\left|\psi_{\omega_{23}}\right\rangle$ have photon numbers bounded form below at two and one, respectively, and we explain it analytically in [56]. $\left\langle\psi_{\omega_{4}}|\dot{H}| \psi_{2+}^{\prime}\right\rangle / \omega^{2} \approx 0$ and decreases with $\left|\Delta_{1}-\Delta_{2}\right| / g$.

On the other hand, the gap between $\left|\psi_{2+}\right\rangle$ and its closest eigenstates other than $\psi_{\omega_{2,3,4}}$ is $\Delta_{1}-\Delta_{2}$, which can be chosen as $\omega$ at $g=0$ provided $\Delta_{1}+\Delta_{2}=\omega$, as shown in Fig. 2(a), while normally the energy gap between the most adjacent levels is much smaller. Although $\left|W_{M} \psi_{B}\right\rangle$ is obtained once $\Delta_{1}=\Delta_{2}$ and $g \neq 0$, we find the adiabatic speed will be much faster if $g$ is increased to the ultrastrong coupling regime. All these factors make the adiabatic evolution quite fast.

We have also considered the parameter fluctuation around $g_{i 1}=g_{i 2}$ and $\Delta_{2}=\omega-\Delta_{1}$ [56] during the adiabatic process. The fidelity $F_{M}=\left|\left\langle W_{M} \psi_{B} \mid \psi(T)\right\rangle\right|^{2}$ is quite robust against such fluctuations. There are possibly two reasons. First, $\left|\left\langle\psi_{2+} \mid \psi_{2+}^{\prime}\right\rangle\right|^{2} \approx 1$, where $\left|\psi_{2+}^{\prime}\right\rangle$ is the deviated special dark state under parameter fluctuations. Second, $\left\langle\psi_{E=\omega}|\dot{H}| \psi_{2+}\right\rangle=0$ reduces to $\left\langle\psi_{\omega_{4}}|\dot{H}| \psi_{2+}^{\prime}\right\rangle / \omega^{2} \approx$ 0 or even vanishes under deviation of parameters [56].

Catch and release of the $W$ state.-On the other hand, just generating a $W$ state inside resonators is not convenient for its transport and detection [49], such that we propose a scheme to store or extract them out on demand in quantum information processes. Our scheme is depicted in Fig. 3(a), where two superconducting qubits (SQs) in the center of the devices are capacitively coupled to $N$ coplanar waveguide resonators (CWRs), which can be described by the two-qubit $M$-mode Rabi model. A detailed description of the superconducting circuit design for the two-qubit twomode Rabi model [see Fig. 3(b)] with independently tunable qubit frequencies and couplings is shown in [56]. Besides, there is an externally variable coupler to modulate the decay rate $\kappa_{c}$ of each CWR through that coupler, such that its photon emission into the connected

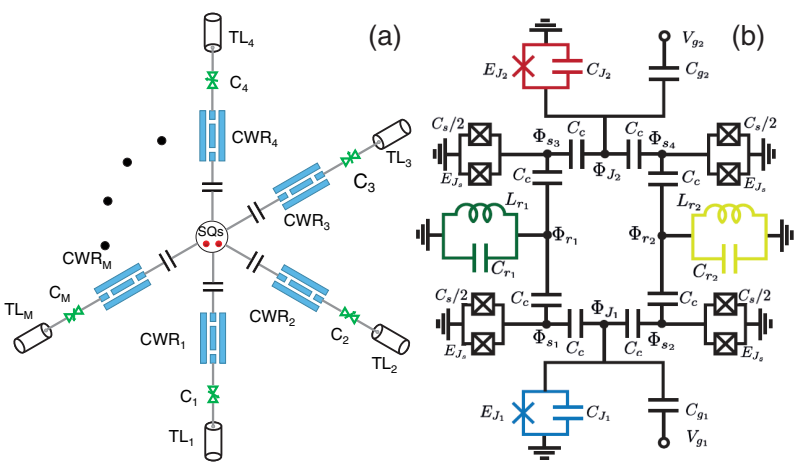

FIG. 3. (a) Schematic setup for the generation and release of the $W$ state: Two SQs are capacitively coupled to $M$ CWRs. Each CWR is connected to a TL through a variable coupler $C$, such that the photon emission rate into the TL is controllable. (b) A superconducting circuit design for the two-qubit two-mode Rabi model [56].

transmission line (TL) is controllable. In current experimental setups, $\kappa_{c}$ can be tuned to be 1000 times the CWR intrinsic decay rate $\kappa_{\text {in }}$ in a few nanoseconds [73], such that we can catch (generate and store) and release the $W$ state on demand.

At the first step, $\left|W_{M} \psi_{B}\right\rangle$ can be generated using the scheme discussed above. Thereafter, the two-qubit Bell state is decoupled from the CWRs, hence $\left|W_{M}\right\rangle$ is naturally stored in resonators for any $g_{i}=g_{i j}$. After a desired time $\tau$, we turn on the coupling $\kappa_{c}$ and therefore the $W$ state is released into the transmission lines. With both $\kappa_{c}$ and intrinsic dissipation included, we use a Lindblad master equation to present the numerical simulation (see Supplemental Material [56] for brief description, which includes Refs. [74,75]) in Fig. 4. The dissipation rates for each CWR are set to be equal, ensuring the emission rate into the $i$ th $\mathrm{TL} \kappa_{c} \operatorname{Tr}\left(a_{i}^{\dagger} a_{i} \rho\right)$ proportional to $g_{i}^{2}$. The time
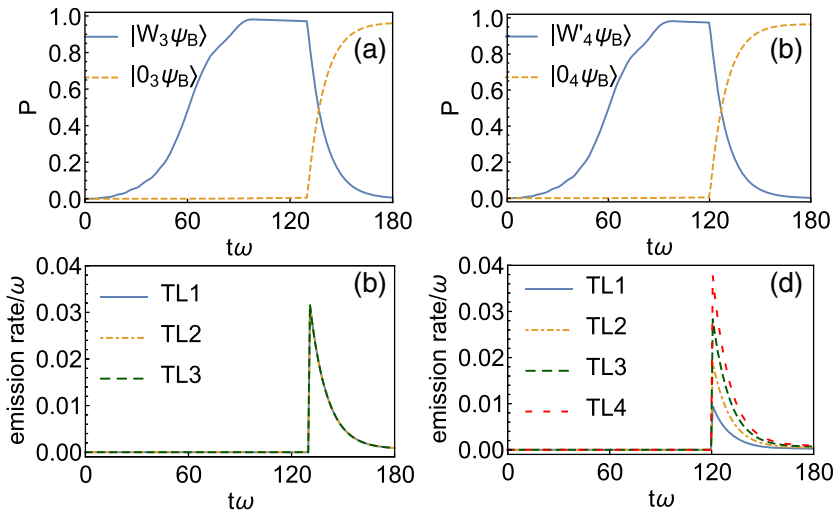

FIG. 4. Numerical simulation for the catch and release of the prototype three-mode $W$ state $\left|W_{3}\right\rangle=(1 / \sqrt{3})(|100\rangle+|010\rangle+$ $|001\rangle$ ) (left panel) and the four-mode $W$ state $\left|W_{4}^{\prime}\right\rangle=$ $(1 / \sqrt{10})(|1000\rangle+\sqrt{2}|0100\rangle+\sqrt{3}|0010\rangle+2|0001\rangle) \quad$ (right panel). (a) and (b) Population of different states inside resonators. (c) and (d) Emission rates into transmission lines. 

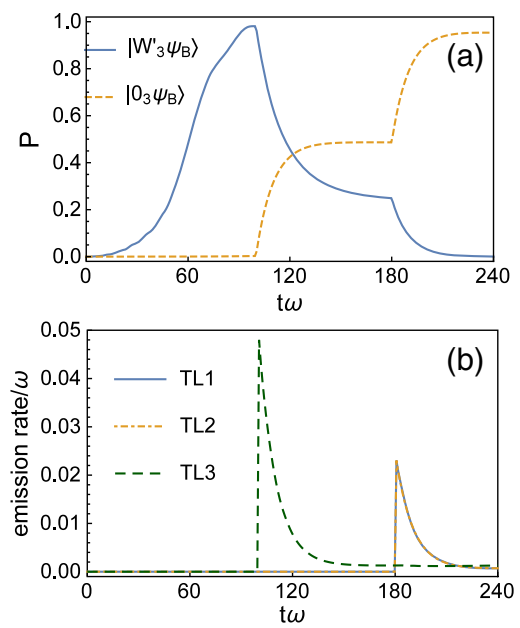

FIG. 5. Numerical simulation for the catch and release of the perfect three-mode $W$ state $\left|W_{3}^{\prime}\right\rangle=\frac{1}{2}(|100\rangle+|010\rangle+\sqrt{2}|001\rangle)$ for quantum teleportation. (a) Population of different states inside resonators. (b) Emission rates into transmission lines.

cost for generating $\left|W_{3}\right\rangle$ and $\left|W_{4}^{\prime}\right\rangle$ is still $100 \omega^{-1}$, but the fidelity is reduced to $98 \%$.

However, the prototype $W$ state $\left|W_{3}\right\rangle=(1 / \sqrt{3})(|100\rangle+$ $|010\rangle+|001\rangle)$ cannot be used to perform quantum teleportation, while the so-called perfect $W$ state $\left|W_{3}^{\prime}\right\rangle=$ $\frac{1}{2}(|100\rangle+|010\rangle+\sqrt{2}|001\rangle)$ can [39]. This state can be easily generated by tuning $g_{3}=\sqrt{2} g_{2}=\sqrt{2} g_{1}$, as shown in Fig. 5(a). The first two qubits are kept by Alice while the third one is sent to Bob to carry out the remote communication. If the distance $L$ between them is known, we can delay the emission of the $W$ state into the first two transmission lines by $L / c$, where $c$ is the speed of light, to assure they receive the qubits at the same time, which will increase the communication security. The corresponding numerical simulation is shown in Fig. 5(b).

Conclusions.-All Fock states are excited by the dipole interaction in the ultrastrong coupling regime of the quantum Rabi model. Therefore, although the operation can be faster, it seems impossible to construct any kind of single photon state deterministically. However, we find that for the MMQRM, there exist special dark eigenstates consisting of only vacuum and single photon multimode $W$ states for the photon part in the whole coupling regime with constant energy. Accordingly, we propose a unified and deterministic scheme to adiabatically generate arbitrary $W$ states using the special dark state solution to the two-qubit $M$-mode quantum Rabi model, being able to take advantages of the ultrastrong coupling and avoid its dynamical complexities. Because of their peculiarities, the time cost according to the current circuit QED technology (33 ns with nonadiabatic error $0.1 \%$ and $21.9 \mathrm{~ns}$ with nonadiabatic error $1 \%$ ) is comparable to the recently reported fastest two-qubit gate (30-45 ns) [55]. Optimization of the adiabatic path could further accelerate the adiabatic process in our protocol. Moreover, the generated $W$ states can be released into the transmission lines on demand, which is illustrated to be useful in quantum information processing. It is interesting to explorer similar uses of other special dark states and fast protocols in quantum information using the MMQRM, with its experimental feasibility provided here. Numerical results show there are special solutions to the MMQRM with photon numbers bounded from below and we have explained its existence analytically, but a rigorous proof is still needed.

J.P. is thankful to Jian Li for helpful discussion. We gratefully acknowledge use of the open source PYTHON numerical packages NumPy, and QuTiP [76]. This work was supported by the National Natural Science Foundation of China (11704320), Natural Science Foundation of Hunan Province, China (2018JJ3482), the National Basic Research Program of China (2015CB921103), the Program for Changjiang Scholars and Innovative Research Team in University (No. IRT13093), the funding from Spanish Government PGC2018-095113-B-I00, PID2019-104002GB-C21 and PID2019-104002GB-C22 (MCIU/AEI/FEDER, UE), Basque Government IT986-16, as well as from Quantum Microwave Communication and Sensing (820505) and OpenSuperQ (820363) of the EU Flagship on Quantum Technologies, EU FET Open Grant Quromorphic (828826), Electronic-Photonic Integrated Quantum Simulator (899368), and Shanghai Science and Technology Commission of Shanghai Municipality (Grant No. 2019SHZDZX01-ZX04).

*jpeng@xtu.edu.cn

tenr.solano@gmail.com

†1lamata@us.es

[1] I. I. Rabi, Phys. Rev. 49, 324 (1936); 51, 652 (1937).

[2] E. T. Jaynes and F. W. Cummings, Proc. IEEE 51, 89 (1963).

[3] D. Braak, Phys. Rev. Lett. 107, 100401 (2011).

[4] Q.-H. Chen, C. Wang, S. He, T. Liu, and K.-L. Wang, Phys. Rev. A 86, 023822 (2012).

[5] Y.-Y. Zhang and Q.-H. Chen, Phys. Rev. A 91, 013814 (2015).

[6] L. W. Duan, Y.-F. Xie, D. Braak, and Q.-H. Chen, J. Phys. A 49, 464002 (2016).

[7] Q. T. Xie, H. H. Zhong, M. T. Batchelor, and C. H. Lee, J. Phys. A 50, 113001 (2017).

[8] R. Puebla, M.-J. Hwang, J. Casanova, and M. B. Plenio, Phys. Rev. Lett. 118, 073001 (2017).

[9] L. Cong, X.-M. Sun, M. Liu, Z.-J. Ying, and H.-G. Luo, Phys. Rev. A 99, 013815 (2019).

[10] X.-M. Sun, L. Cong, H.-P. Eckle, Z.-J. Ying, and H.-G. Luo, Phys. Rev. A 101, 063832 (2020).

[11] S. Felicetti and A. Le Boité, Phys. Rev. Lett. 124, 040404 (2020). 
[12] D. Lv, S. An, Z. Liu, J.-N. Zhang, J. S. Pedernales, L. Lamata, E. Solano, and K. Kim, Phys. Rev. X 8, 021027 (2018).

[13] Y. Lu and C. Wang, Quantum Inf. Process. 15, 4347 (2016).

[14] T. Werlang, A. V. Dodonov, E. I. Duzzioni, and C. J. Villas-Bôas, Phys. Rev. A 78, 053805 (2008).

[15] J. Casanova, G. Romero, I. Lizuain, J. J. García-Ripoll, and E. Solano, Phys. Rev. Lett. 105, 263603 (2010).

[16] J. S. Pedernales, I. Lizuain, S. Felicetti, G. Romero, L. Lamata, and E. Solano, Sci. Rep. 5, 15472 (2015).

[17] L. Lamata, Sci. Rep. 7, 43768 (2017).

[18] D. D. Sedov, V. K. Kozin, and I. V. Iorsh, Phys. Rev. Lett. 125, 263606 (2020).

[19] E. K. Irish, Phys. Rev. Lett. 99, 173601 (2007).

[20] A. Blais, R.-S. Huang, A. Wallraff, S. M. Girvin, and R. J. Schoelkopf, Phys. Rev. A 69, 062320 (2004).

[21] X.-Y. Lü, G.-L. Zhu, L.-L. Zheng, and Y. Wu, Phys. Rev. A 97, 033807 (2018).

[22] I. Thanopulos, E. Paspalakis, and Z. Kis, Chem. Phys. Lett. 390, 228 (2004).

[23] G. Romero, D. Ballester, Y. M. Wang, V. Scarani, and E. Solano, Phys. Rev. Lett. 108, 120501 (2012).

[24] R. Barends, C. M. Quintana, A. G. Petukhov, Y. Chen, D. Kafri, K. Kechedzhi et al., Phys. Rev. Lett. 123, 210501 (2019).

[25] A. Barenco, C. H. Bennett, R. Cleve, D. P. DiVincenzo, N. Margolus, P. Shor, T. Sleator, J.A. Smolin, and H. Weinfurter, Phys. Rev. A 52, 3457 (1995).

[26] M. Hua, M.-J. Tao, and F.-G. Deng, Phys. Rev. A 90, 012328 (2014).

[27] Y.-H. Kang, Y.-H. Chen, Q.-C. Wu, B.-H. Huang, J. Song, and Y. Xia, Sci. Rep. 6, 36737 (2016).

[28] M. Lu, Y. Xia, J. Song, and N. Ba An, J. Opt. Soc. Am. B 30, 2142 (2013).

[29] J. Li and G. S. Paraoanu, New J. Phys. 11, 113020 (2009).

[30] R. Koç, M. Koca1, and H. Tütüncüler, J. Phys. A 35, 9425 (2002).

[31] F. Pan, Y. K. Yao, M.-X. Xie, W.-J. Han, and J. P. Draayer, Commun. Theor. Phys. 48, 53 (2007).

[32] B. R. Judd, J. Phys. C 12, 1685 (1979).

[33] M. O. Scully and M. S. Zubairy, Quantum Optics (Cambridge University Press, Cambridge, England, 1997), Chap. 7.

[34] L. Zhou, Y.-B. Sheng, W.-W. Cheng, L.-Y. Gong, and S.-M. Zhao, J. Opt. Soc. Am. B 30, 71 (2013).

[35] A. A. Gangat, I. P. McCulloch, and G. J. Milburn, Phys. Rev. X 3, 031009 (2013).

[36] Y.-B. Sheng, Y. Ou-Yang, L. Zhou, and L. Wang, Quantum Inf. Process. 13, 1595 (2014).

[37] A. Sharma and A. A. Tulapurkar, Phys. Rev. A 101, 062330 (2020).

[38] N. Kiesel, M. Bourennane, C. Kurtsiefer, H. Weinfurter, D. Kaszlikowski, W. Laskowski, and M. Zukowski, J. Mod. Opt. 50, 1131 (2003).

[39] P. Agrawal and A. Pati, Phys. Rev. A 74, 062320 (2006).

[40] S.-B. Zheng, Phys. Rev. A 74, 054303 (2006).

[41] L. Li and D. Qiu, J. Phys. A 40, 10871 (2007).

[42] L.-H. Yan, Y.-F. Gao, and J.-G. Zhao, Int. J. Theor. Phys. 48, 2445 (2009).
[43] M. Eibl, N. Kiesel, M. Bourennane, C. Kurtsiefer, and H. Weinfurter, Phys. Rev. Lett. 92, 077901 (2004).

[44] M. Menotti, L. Maccone, J. E. Sipe, and M. Liscidini, Phys. Rev. A 94, 013845 (2016).

[45] B. Fang, M. Menotti, M. Liscidini, J. E. Sipe, and V. O. Lorenz, Phys. Rev. Lett. 123, 070508 (2019).

[46] X. B. Zou, K. Pahlke, and W. Mathis, Phys. Rev. A 66, 044302 (2002).

[47] G.-P. Guo, C.-F. Li, J. Li, and G.-C. Guo, Phys. Rev. A 65, 042102 (2002).

[48] Z. J. Deng, M. Feng, and K. L. Gao, Phys. Rev. A 73, 014302 (2006).

[49] G.-C. Guo and Y.-S. Zhang, Phys. Rev. A 65, 054302 (2002).

[50] Y.-H. Kang, Y.-H. Chen, Q.-C. Wu, B.-H. Huang, J. Song, and Y. Xia, Sci. Rep. 6, 36737 (2016).

[51] V. M. Stojanović, Phys. Rev. Lett. 124, 190504 (2020).

[52] C. Li and Z. Song, Phys. Rev. A 91, 062104 (2015).

[53] Y.-H. Kang, Y.-H. Chen, Z.-C. Shi, J. Song, and Y. Xia, Phys. Rev. A 94, 052311 (2016).

[54] J. Chen, H. Zhou, C. Duan, and X. Peng, Phys. Rev. A 95, 032340 (2017).

[55] M. A. Rol et al., Phys. Rev. Lett. 123, 120502 (2019).

[56] See Supplemental Material at http://link.aps.org/ supplemental/10.1103/PhysRevLett.127.043604 for the method to obtain the special quasiexact solution to the multiqubit multimode Rabi model, the peculiarities of the special dark state $\left|\psi_{2+}\right\rangle$, stability analysis of the fidelity $\left|\left\langle W_{M} \psi_{B} \mid \psi(T)\right\rangle\right|^{2}$ under parameter fluctuations, demonstration of the circuit design for the implementation of the twoqubit two-mode quantum Rabi model, the Lindblad master equation we used for numerical simulation and the adiabatic path used to generate $\left|W_{M} \psi_{B}\right\rangle$.

[57] J. Peng, Z.Z. Ren, D. Braak, G. J. Guo, Guoxing Ju, X. Zhang, and X. Y. Guo, J. Phys. A 47, 265303 (2014).

[58] J. Peng, Z. Z. Ren, H. T. Yang, G. J. Guo, X. Zhang, G. X. Ju, X. Y. Guo, C. S. Deng, and G. L. Hao, J. Phys. A 48, 285301 (2015).

[59] J. Peng, C. X. Zheng, G. J. Guo, X. Y. Guo, X. Zhang, C. S. Deng, G. X. Ju, Z.Z. Ren, L. Lamata, and E. Solano, J. Phys. A 50, 174003 (2017).

[60] M. D. Hutchings, J. B. Hertzberg, Y. Liu, N. T. Bronn, G. A. Keefe, M. Brink, J. M. Chow, and B. L. T. Plourde, Phys. Rev. Applied 8, 044003 (2017).

[61] P. Krantz, M. Kjaergaard, F. Yan, T. P. Orlando, S. Gustavsson, and W.D. Oliver, Appl. Phys. Rev. 6, 021318 (2019).

[62] A. Blais, A. L. Grimsmo, S. M. Girvin, and A. Wallraff, Rev. Mod. Phys. 93, 025005 (2021).

[63] J. M. Fink, R. Bianchetti, M. Baur, M. Göppl, L. Steffen, S. Filipp, P. J. Leek, A. Blais, and A. Wallraff, Phys. Rev. Lett. 103, 083601 (2009).

[64] Y. P. Zhong et al., Nature (London) 590, 571 (2021).

[65] Y. Lu et al., Phys. Rev. Lett. 119, 150502 (2017).

[66] S. J. Srinivasan, A. J. Hoffman, J. M. Gambetta, and A. A. Houck, Phys. Rev. Lett. 106, 083601 (2011).

[67] R. Barends, J. Kelly, A. Megrant, A. Veitia, D. Sank et al., Nature (London) 508, 500 (2014). 
[68] M. Kjaergaard, M. E. Schwartz, J. Braumüller, P. Krantz, J. I.-J. Wang, S. Gustavsson, and W. D. Oliver, Annu. Rev. Condens. Matter Phys. 11, 369 (2020).

[69] J. M. Martinis and M. R. Geller, Phys. Rev. A 90, 022307 (2014).

[70] Y. Chen, C. Neill, P. Roushan, N. Leung, M. Fang, and R. Barends et al., Phys. Rev. Lett. 113, 220502 (2014).

[71] P. Ehrenfest, Ann. Phys. (Leipzig) 356, 327 (1916).

[72] M. Born and V. Fock, Z. Phys. 51, 165 (1928).
[73] Y. Yin, Y. Chen, D. Sank, P. J. J. O’Malley, T. C. White, R. Barends et al., Phys. Rev. Lett. 110, 107001 (2013).

[74] F. Beaudoin, J. M. Gambetta, and A. Blais, Phys. Rev. A 84, 043832 (2011).

[75] A. Ridolfo, M. Leib, S. Savasta, and M. J. Hartmann, Phys. Rev. Lett. 109, 193602 (2012).

[76] J. R. Johansson, P. D. Nation, and F. Nori, Comput. Phys. Commun. 184, 1234 (2013). 